\title{
Does a history of head and neck cancer affect outcome of endoscopic submucosal dissection for superficial esophageal squamous cell carcinoma?
}

\section{다 (1) $(2)$}

\author{
Authors \\ Renata Nobre Moura1', Vitor Nunes Arantes², Tarso Magno Leite Ribeiro², Roberto Gardone Guimarães², Joel \\ Fernandez de Oliveira ${ }^{1}$, Marco Aurélio Vamondes Kulcsar ${ }^{1}$, Rubens Antonio Aissar Sallum ${ }^{1}$, Ulysses Ribeiro-Junior ${ }^{1}$, \\ Fauze Maluf-Filho ${ }^{1}$
}

Institutions

1 Department of Gastroenterology, Cancer Institute, Hospital das Clinicas of University of Sao Paulo, Sao Paulo, Brazil

2 Alfa Institute of Gastroenterology, Hospital de Clínicas, Federal University of Minas Gerais, Belo Horizonte, Brazil

submitted 3.9.2019

accepted after revision 17.2 .2020

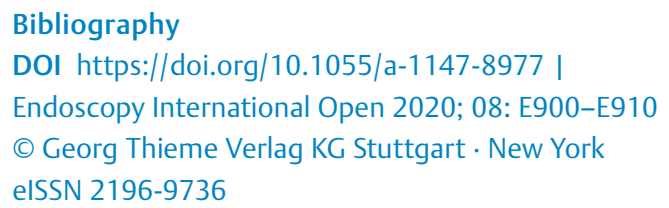

Corresponding author

Renata Nobre Moura, Division of Gastroenterology, Sao Paulo Cancer Institute, Av. Dr. Arnaldo, 251, São PauloBrazil 01246-000, Brazil

Fax: +551138877593

renata.nobre@fm.usp.br

\section{ABSTRACT}

Background and study aims Esophageal squamous cell carcinoma (ESCC) is the most common secondary tumor in patients with head and neck squamous cell cancer
(HNSCC). Currently, endoscopic submucosal dissection (ESD) is the preferred approach to manage superficial ESCC, however, it remains to be elucidated whether patients with HNSCC and early ESCC managed by ESD have different outcomes.

Patients and methods We retrospectively analyzed esophageal ESD for early ESCC from September 2009 to September 2017 and the following variables: demographics, tumor and specimen size, Paris classification, location, en bloc and $\mathrm{R} 0$ resection rates, overall survival (OS) and adverse events (AEs). To reduce selection bias, propensity score matching was applied to compare the results.

Results Eighty-nine ESDs were performed in 81 consecutive patients (47 with HNSCC and 34 without HNSCC). Patients with HNSCC who developed superficial ESCC were found to be younger and to refer a more frequent history of alcohol ingestion and smoking. There was no difference in lesion size, number of lesions, procedure time, en bloc resection rate, $\mathrm{R} 0$ resection rate, local recurrence and adverse event rate between the two groups. The histological depth of invasion for patients with HNSCC was significantly shallower before $(P=0.016)$ and after $(P=0.047)$ matching. The overall survival rate was similar in both groups.

Conclusions Patients with HNSCC have earlier detection of ESCC, probably due to endoscopic screening. Previous history of chemoradiation and surgery for HNSCC does not affect procedure time, AEs and OS.

\section{Introduction}

Patients with head and neck squamous cell cancer (HNSCC) frequently develop a secondary tumor, which can occur in the upper gastrointestinal tract, head and neck or lungs, with incidences ranging from $16 \%$ to $36 \%[1,2]$. Esophageal squamous cell carcinoma (ESCC) is the most common secondary tumor in these patients with a reported mean incidence of $5 \%$ to $15 \%$ and poor prognosis due to a late diagnosis in $90 \%$ of cases [3] being the main cause of treatment failure and mortality, responsible for more than one-third of deaths in patients with HNSCC [4]. When ESCC is diagnosed early, the expected 5-year survival rate is $85 \%$ to $100 \%[4,5]$. Hence, undertaking an esophageal cancer screening protocol in high-risk patients such as individuals with HNSCC may be justified and improve prognosis. At our Institutions, all patients with HNSCC (except those with Stage IV disease), despite the absence of esophageal symptoms, are invited to undertake an endoscopic screening examination. At both centers, a meticulous white-light examination (WLE) is followed by virtual chromoendoscopy and lugol 
staining. This protocol has yielded a $7 \%$ to $12 \%$ finding of ESCC, and interestingly in over $70 \%$ of the patients, esophageal neoplastic lesions were superficial and, therefore, suitable for endoscopic resection $[6,7]$.

Endoscopic techniques have been developed for curative resection of superficial neoplasms of the esophagus, such as endoscopic mucosal resection (EMR) and endoscopic submucosal dissection (ESD). Currently, ESD is considered the preferred approach to manage superficial ESCC, enabling accurate en bloc resection with a lower recurrence rate and improved survival [8-11]. Until 2014, 15 studies had been published specifically on ESD for ESCC, totaling 970 lesions. The en bloc resection rate was $99 \%(83.3 \%-100 \%)$, R0 resection rate, $82.8 \%$ (78\%-100\%), and curative resection rate, 75.6\% (69\%-100\%), with a local recurrence rate of $0.3 \%(0 \%-2.6 \%)$ and no mortality $[8,12]$.

It remains to be elucidated whether patients with HNSCC who develop ESCC and are managed by ESD have different clinical outcomes compared with patients without HNSCC. In patients with HNSCC, the so-called "field cancerization phenomena," caused by exposure to environmental carcinogens, most notably alcohol and tobacco, would lead to genetic alterations and, subsequently, development of multiple and independent tumors. This association is related to poor survival rates for both primary and secondary tumors [13]. Furthermore, the effects of radiotherapy might hinder endoscopic resection due to scarring or fibrosis and potentially increase adverse event (AE) rate. However, to date, no comparative studies have been performed to evaluate the outcome of ESCC patients with HNSCC managed by ESD.

This study aimed to compare ESD outcomes of both sporadic and HNSCC-associated ESCC. Endpoints were en-bloc resection rate, $\mathrm{R} 0$ resection rate, curative resection rate, survival and $\mathrm{AE}$ rate.

\section{Patients and methods}

This was a retrospective analysis of patients who had undergone ESD for early ESCC from September 2009 to September 2017 at two academic tertiary centers in Brazil. Both centers are major referral Institutions to manage HNSCC patients in Brazil health care public system. Included criteria was superficial ESCC suitable for endoscopic resection by ESD.

Patients were divided into two groups: those with HNSCC (group 1) and those without a previous history of HNSCC (group 2). Clinical information was extracted from a prospectively collected database: demographics, tumor and specimen size, Paris classification, carcinoma location, en bloc resection rate, $R 0$ and curative resection rate, overall survival (OS), local recurrence and AEs. Informed consent for both diagnostic screening EGD and ESD resection was obtained from all patients. Approval was obtained by the Local Ethic Committee of both hospitals. All procedural data on patients and their lesions were collected in a database. When necessary, contact by telephone call was made.

Patients with newly diagnosed HNSCC regardless of previous treatment (surgery or chemoradiation) were submitted to the
Screening Program, which comprises annually EGD with chromoscopy (lugol iodine staining and NBI). Exclusion criteria for the Screening Protocol were previous ESCC, history of esophageal surgery or advanced HNSCC stage according the $7^{\text {th }}$ AJCC TNM classification [14]. After finishing the HNSCC treatment, all patients were advised to continue on the Program to evaluate metachronous ESCC. Patients from the non-NNCSCC group were not submitted to screening endoscopy. They were referred to EGD for the investigation of dyspeptic symptoms.

First, the patients were submitted to conventional WLE with evaluation of oropharynx and hypopharynx under conscious sedation. After washing the esophageal lumen, a meticulous WLE observation was carried out searching for slight color changes, loss of normal vascular pattern or surface irregularities. The second phase involved the use of virtual chromoendoscopy such as NBI to evaluate changes in the intraepithelial papillary capillary loops (IPCLs). In the third phase, Lugol's staining was performed by spraying $20 \mathrm{cc}$ of a $1 \%$ Lugol's solution at esophageal mucosa. Unstained lesions that presented a pink-color sign were considered neoplastic or suspicious and biopsies were taken for histological assessment.

Indication for endoscopic resection was based on Japanese Esophageal Society Guidelines as follows: superficial lesions limited to mucosal layer (T1a) or possible slight infiltration of superficial submucosa, regardless of size or circumferential involvement, were considered suitable for ESD.

Assessment for preoperative diagnosis of invasion and endoscopic resection feasibility was based on detailed endoscopic evaluation with virtual chromoendoscopy and magnifying endoscopy when available. In selected cases endoscopic ultrasound (EUS) was performed. Thorax computed tomography was part of the preoperative staging protocol. All patients had diagnosis confirmation with histological assessment of endoscopic biopsies.

ESD was performed by two experienced endoscopists (F.M.F and V.A) previously trained in Japan, utilizing a needle-type knife (Flush-Knife BT, Fujifilm Co., Japan), following the basic steps: marking, injection, incision, submucosal dissection, and revision of ESD site.

When ESD was considered curative, first EGD control with WLE examination was scheduled at 3 months aiming to check ESD site healing and any signs of residual tumor. Thereafter, annual EGD control with virtual chromoendoscopy and lugol staining was recommended. After circumferential ESD or semicircumferential resection producing a defect over $75 \%$ of the circumference, preventive stricture measures were undertaken with postoperative administration of oral or injected steroids. Those patients underwent first EGD control at the end of treatment (4 weeks) and endoscopic dilation was performed if dysphagia symptoms or post-ESD stricture were noted.

Tumor size, depth of invasion, lymphovascular invasion, grade of differentiation, and resection margins were histopathologically examined by expert gastrointestinal pathologists, according to the Vienna Classification [15]. 


\section{Definitions}

En bloc resection was achieved when the whole lesion (including the markings) was removed in one piece. $R 0$ resection was defined histologically when margins were negative for neoplastic cells. Endoscopic resection was considered curative when the tumor was resected en bloc, with free vertical margin (R0), pathologically invasion depth was limited to muscularis mucosa (MM) and there was no lymphovascular involvement [16]. Patients with non-curative ESD were referred to additional treatments indicated within the scope of multidisciplinary decision (e.g., esophagectomy with lymph node dissection or radiation therapy with or without chemotherapy).

Procedure time, defined as the time from the marking of the lesion to the completion of lesion resection, was measured in minutes.

Perforation was recognized when mediastinal connective tissue or subcutaneous emphysema was observed during the procedure, or radiographic evidence of free air on chest $x$-ray after ESD. Chest $x$-ray was performed after ESD only when a patient was referred for thoracic pain or dyspnea. Perforation was further classified in patients treated endoscopically without clinical repercussion and those who required thoracic drainage or any other surgical approach.

Penetrating blood vessels were sealed using the knife itself or coagulation forceps. Delayed bleeding was defined as decreased blood hemoglobin level $>2 \mathrm{~g} / \mathrm{dL}$ accompanied by hematemesis or melena.

Postoperative stricture was defined as dysphagia and the inability to pass a standard endoscope, requiring treatment such as endoscopic dilation. Tumor recurrence was defined as a finding of neoplastic cells at the scar site in a follow-up EGD control. Metachronous tumor was defined as the occurrence of a novel neoplastic lesion in the esophagus $>1 \mathrm{~cm}$ apart from the scar site occurring more than 1 year after the index endoscopic resection.

\section{Statistical analysis}

Patient characteristics were summarized using descriptive statistics. Categorical variables were presented as absolute and relative frequencies and quantitative data as means and SDs.

Potential differences between the experimental groups were assessed by using the Chi square test or, in case of small sample, the Fisher exact probability test. The Student's $t$ test was used to compare means. This test presupposes a normal distribution, which was verified by the Kolmogorov-Smirnov test. When not normally distributed, the Mann-Whitney nonparametric test was used.

Because of differences between the two groups, propensity score matching (PSM) was applied to compare the results. This test aims to reduce or eliminate selection bias in observational studies, which is a useful strategy when random allocation is not possible. PSM employs a predicted probability of group membership based on observed predictors obtained from logistic regression. In this study, PSM was used to match the groups (patients without HNSCC who have similar characteristics to those with HNSCC). Selection of patients was done by the nearest neighbor matching with caliper of 0.15 to prevent poor matching. Variables included in the matching model were alcohol consumption, smoking, age, and Paris Classification.

Kaplan-Meier curves were constructed to analyze rates of OS and cancer-related survival.

For all analyses, $P<5 \%$ was considered statistically significant.

Statistical analyses were conducted using SPSS software 20.0 and STATA 12.

\section{Results}

From September 2009 to September 2017, 848 esophageal cancers were diagnosed as shown in > Fig.1. From among them, 89 esophageal ESDs to resect superficial ESCC were carried out in 81 consecutive patients. Forty-seven of them (58\%) had a previous history of HNSCC and had their tumor detected due to the endoscopic screening protocol. The remaining patients did not have dysphagia nor weight loss and were diagnosed during routine endoscopy motivated by dyspeptic symptoms. The male-to-female ratio was $3.5: 1$ and the average age was 62.2 years (range $41-84$ years). HNSCC subjects who developed superficial ESCC were found to be significantly younger (mean age $58.8 \pm 9.5$ vs. $67 \pm 9.2 ; P<0.001$ ) and more likely to consume alcohol and to smoke $(91.5 \%$ vs. $58.8 \% P<0.001$ and $93.6 \%$ vs. $67.6 \% P=0.002$, respectively).

Median specimen size was $3.5 \mathrm{~cm}$, with no statistical difference between the two groups. Most of the tumors were located in the middle esophagus (66\%). In the HNSCC group, $84.3 \%$ of lesions was classified as flat type (Paris 0 -IIB), which was statistically different than the other group $(P=0.023)$. Demographics and clinicopathological characteristics are shown in $>$ Table 1.

In the group with HNSCC, the location of the index tumor was oral cavity in $48.9 \%$, oropharynx in $23.4 \%$, larynx in $19.1 \%$, and hypopharynx in $6.3 \%$ of the patients. As regards HNSCC stage, 25 patients $\left(53.1 \%\right.$ ) had stage III disease ( $7^{\text {th }}$ AJCC TNM classification). Forty-one patients (87.2\%) had previously received chemoradiation and 28 had undergone surgery for the primary cancer.

During the Screening Program, 187 EGDs were performed in 81 patients (mean of 2.3 exams per patient). Esophageal cancer was synchronous to head and neck cancer in 12 patients (25.5\%). In the cases of metachronous ESCC, the mean interval was 21 months.

Overall, the mean procedure time, resection and $A E$ rates were 118 minutes, $97.7 \%$ and $10.1 \%$, respectively, with no difference between the two groups. In the HNSCC group, esophageal cancer was more frequently found limited to the mucosal layer ( $88.2 \%$ vs. $65.8 \%, P=0.016)$. ESD outcomes are shown in - Table 2.

R0 resection was possible in 81 lesions (91\%) in 74 patients $(91.3 \%)$, with no difference between the patients with or without HNSCC (92.2\% vs $89.5 \%$ respectively, $P=0.719)$. The curative resection rate was $78.4 \%$ in group 1 (HNSCC) and $57.8 \%$ in group $2(P=0.037)$. Reasons for non-curative resection lesions 


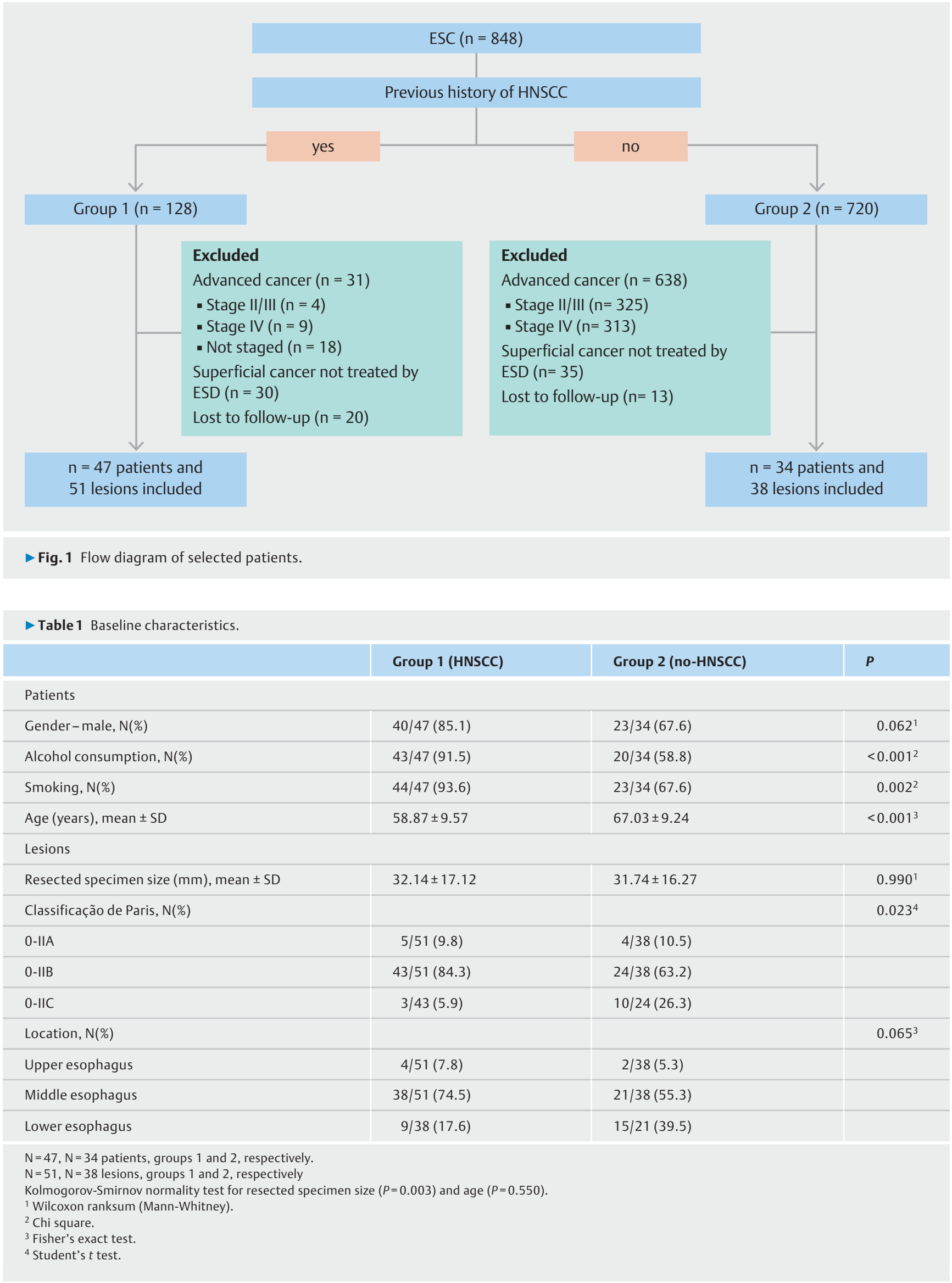


- Table2 ESD outcomes.

\begin{tabular}{|c|c|c|c|}
\hline & Group 1 (HNSCC) & Group 2 (no-HNSCC) & $P$ \\
\hline \multicolumn{4}{|l|}{ Lesions } \\
\hline Resected specimen size $(\mathrm{mm})$, mean \pm SD & $32.14 \pm 17.12$ & $31.74 \pm 16.27$ & $0.990^{1}$ \\
\hline Circumference, $\mathrm{N}(\%)$ & & & $0.902^{2}$ \\
\hline$<50 \%$ & $13 / 51(25.5)$ & $12 / 38(31.6)$ & \\
\hline $50 \%-75 \%$ & $21 / 51(41.2)$ & $13 / 38(34.2)$ & \\
\hline $75 \%-100 \%$ & $12 / 51(23.5)$ & $9 / 38(23.7)$ & \\
\hline $100 \%$ & $5 / 51(9.8)$ & $4 / 38(10.5)$ & \\
\hline Procedure time $(\mathrm{min})$, mean \pm SD & $107.42 \pm 40.15$ & $102.03 \pm 36.08$ & $0.531^{3}$ \\
\hline Histological depth of invasion, N(\%) & & & $0.016^{4}$ \\
\hline Intramucosa & $45 / 51(88.2)$ & $25 / 38(65.8)$ & \\
\hline Superficial submucosa & $5 / 51(9.8)$ & $7 / 38(18.4)$ & \\
\hline Deep submucosa & $1 / 51(2.0)$ & $6 / 38(15.8)$ & \\
\hline En bloc ressection & $50 / 51(98.0)$ & $37 / 38(97.4)$ & $1.000^{1}$ \\
\hline R0 ressection & $47 / 51(92.2)$ & $34 / 38(89.5)$ & $0.719^{1}$ \\
\hline Curative resection & $40 / 51(78.4)$ & $22 / 38(57.9)$ & $0.037^{3}$ \\
\hline \multicolumn{4}{|l|}{ Procedure-related complications } \\
\hline Bleeding & $0 / 47(0.0)$ & $0 / 38(0.0)$ & - \\
\hline Perforation & $2 / 50(4.0)$ & $1 / 38(2.6)$ & $1.000^{4}$ \\
\hline Stricture (estenose) & $17 / 51(33.3)$ & $8 / 38(21.1)$ & $0.202^{2}$ \\
\hline Tumor Local recurrence & $1 / 51(2.0)$ & $1 / 38(2.6)$ & $1.000^{4}$ \\
\hline Metachronous tumor & $10 / 51(19.6)$ & $7 / 38(18.4)$ & $0.888^{2}$ \\
\hline Synchronous tumor & $4 / 51(7.8)$ & $2 / 38(5.3)$ & $1.000^{4}$ \\
\hline \multicolumn{4}{|l|}{ Patients } \\
\hline Adjuvant therapy & $7 / 46(15.2)$ & $9 / 34(26.5)$ & $0.214^{2}$ \\
\hline Mortality & $17 / 47(36.2)$ & $9 / 34(26.5)$ & $0.356^{2}$ \\
\hline $\begin{array}{l}\text { ESD, endoscopic submucosal dissection; HNSC, } \\
\mathrm{N}=47, \mathrm{~N}=34 \text { patients, groups } 1 \text { and } 2 \text {, respecti } \\
\mathrm{N}=51, \mathrm{~N}=38 \text { lesions, groups } 1 \text { and } 2 \text {, respectiv } \\
\text { Kolmogorov-Smirnov normality test for resecte } \\
{ }^{1} \text { Wilcoxon ranksum (Mann-Whitney). } \\
{ }^{2} \text { Chi square. } \\
{ }^{3} \text { Student's } t \text { test. } \\
{ }^{4} \text { Fisher's exact test. }\end{array}$ & $\begin{array}{l}(P=0.003) \text { and procec } \\
(P \text { amous cell carcinor }\end{array}$ & deviation. & \\
\hline
\end{tabular}

were as follows: SM invasion (19), positive vertical margins (3), lymphovascular invasion (4), piecemeal resection (1).

Of the 27 patients with non-curative endoscopic resection, 16 underwent adjuvant treatment (esophagectomy $n=5$, chemoradiation $n=11$ ). The remaining patients were placed under observation because of severe comorbities, advanced age or patient refusal of additional treatment. Those who achieved curative resection underwent annual endoscopic surveillance.

AEs occurred in 28 cases (perforation $=3$; stricture $=25$ ), with no difference between the groups. In the group with HNSCC, one patient had a perforation during the procedure and developed pneumothorax and pneumomediastinum. The patient was managed by clipping, pigtail thorax drainage, enteral feeding and antibiotics. Another patient had a delayed perforation and was successfully managed with enteral feeding and antibiotics. In the group of non-HNSCC, one patient developed subcutaneous emphysema after the procedure managed by enteral feeding and antibiotics. Clinically relevant bleeding was not observed in this cohort.

All 25 patients who developed stricture (group $1=17$, group $2=8$ ) were managed by endoscopic balloon dilation. Cases with mucosal defect over $75 \%$ of the circumference received oral 
- Table 3 ESD outcomes before and after PSM.

\begin{tabular}{|c|c|c|c|c|c|c|}
\hline & \multicolumn{3}{|l|}{ Original cohort } & \multicolumn{3}{|l|}{ Matched cohort } \\
\hline & $\begin{array}{l}\text { Group } 1 \\
\text { (HNSCC) }\end{array}$ & $\begin{array}{l}\text { Group } 2 \\
\text { (no-HNSCC) }\end{array}$ & $P$ & $\begin{array}{l}\text { Group } 1 \\
\text { (HNSCC) }\end{array}$ & $\begin{array}{l}\text { Group } 2 \\
\text { (no-HNSCC) }\end{array}$ & $P$ \\
\hline \multicolumn{7}{|l|}{ Lesions } \\
\hline Resected specimen size $(\mathrm{mm})$, mean \pm SD & $32.14 \pm 17.12$ & $31.74 \pm 16.27$ & $0.990^{1}$ & $31.26 \pm 16.89$ & $23.04 \pm 7.99$ & $0.012^{1}$ \\
\hline Circunference, $\mathrm{N}(\%)$ & & & $0.902^{2}$ & & & $0.147^{3}$ \\
\hline$<50 \%$ & $25.5 \%$ & $31.6 \%$ & & $27.7 \%$ & $29.8 \%$ & \\
\hline $50 \%-75 \%$ & $41.2 \%$ & $34.2 \%$ & & $40.4 \%$ & $57.4 \%$ & \\
\hline $75 \%-100 \%$ & $23.5 \%$ & $23.7 \%$ & & $25.5 \%$ & $10.6 \%$ & \\
\hline $100 \%$ & $9.8 \%$ & $10.5 \%$ & & $6.4 \%$ & $2.1 \%$ & \\
\hline Procedure time $(\min )$, mean \pm SD & $107.42 \pm 40.15$ & $102.03 \pm 36.08$ & $0.531^{4}$ & $106.34 \pm 39.87$ & $102.02 \pm 41.85$ & $0.610^{4}$ \\
\hline Histological depth of invasion, $\mathrm{N}(\%)$ & & & $0.016^{4}$ & & & $0.047^{4}$ \\
\hline Intramucosa & $88.2 \%$ & $65.8 \%$ & & $87.2 \%$ & $66.0 \%$ & \\
\hline Superficial submucosa & $9.8 \%$ & $18.4 \%$ & & $10.6 \%$ & $23.4 \%$ & \\
\hline Deep submucosa & $2.0 \%$ & $15.8 \%$ & & $2.1 \%$ & $10.6 \%$ & \\
\hline En bloc ressection & $98.0 \%$ & $97.4 \%$ & $1.000^{3}$ & $97.9 \%$ & $100.0 \%$ & $1.000^{3}$ \\
\hline R0 ressection & $92.2 \%$ & $89.5 \%$ & $0.719^{3}$ & $91.5 \%$ & $95.7 \%$ & $0.677^{3}$ \\
\hline Curative resection & $78.4 \%$ & $57.9 \%$ & $0.037^{2}$ & $76.6 \%$ & $66.0 \%$ & $0.254^{2}$ \\
\hline \multicolumn{7}{|l|}{ Procedure related complications } \\
\hline Bleeding & $0.0 \%$ & $0.0 \%$ & - & $0.0 \%$ & $0.0 \%$ & - \\
\hline Perforation & $4.0 \%$ & $2.6 \%$ & $1.000^{3}$ & $4.3 \%$ & $0.0 \%$ & $0.495^{3}$ \\
\hline Stricture & $33.3 \%$ & $21.1 \%$ & $0.202^{2}$ & $29.8 \%$ & $19.1 \%$ & $0.203^{2}$ \\
\hline Tumor Local recurrence & $2.0 \%$ & $2.6 \%$ & $1.000^{3}$ & $2.1 \%$ & $0.0 \%$ & $1.000^{3}$ \\
\hline Metachronous tumor & $19.6 \%$ & $18.4 \%$ & $0.888^{2}$ & $19.1 \%$ & $19.1 \%$ & $1.000^{2}$ \\
\hline Synchronous tumor & $7.8 \%$ & $5.3 \%$ & $1.000^{3}$ & $6.4 \%$ & $17.0 \%$ & $0.109^{2}$ \\
\hline \multicolumn{7}{|l|}{ Patients } \\
\hline Adjuvant therapy & $15.2 \%$ & $26.5 \%$ & $0.214^{2}$ & $15.2 \%$ & $17.4 \%$ & $0.778^{2}$ \\
\hline Mortality & $36.2 \%$ & $26.5 \%$ & $0.356^{2}$ & $37.0 \%$ & $21.7 \%$ & $0.109^{2}$ \\
\hline \multicolumn{7}{|c|}{$\begin{array}{l}\text { ESD, endoscopic submucosal dissection; HNSCC, head and neck squamous cell carcinoma; SD, standard deviation; PSM, propensity score matching. } \\
\text { Original cohort: } N=47 \text { and } N=34 \text { patients (group } 1 \text { and } 2 \text {, respectively). } N=51 \text { e } N=38 \text { lesions (group } 1 \text { and } 2 \text {, respectively). } \\
\text { Matched cohort: } N=46 \text { patients (groups } 1 \text { and } 2) . N=47 \text { lesions (groups } 1 \text { and } 2 \text { ) } \\
{ }^{1} \text { Wilcoxon ranksum (Mann-Whitney). } \\
{ }^{2} \text { Chi square. } \\
{ }^{3} \text { Fisher's exact test. } \\
{ }^{4} \text { Student's t test. }\end{array}$} \\
\hline
\end{tabular}

corticoids for 4 weeks (starting at $30 \mathrm{mg} /$ day oral prednisone tapered weekly to $20 \mathrm{mg} /$ day, $10 \mathrm{mg} /$ day and ending at $5 \mathrm{mg} /$ day) or local steroid injection (triamcinolone $100 \mathrm{mg}$ ), according to an ongoing randomized controlled trial.

After propensity score matching (PSM) was performed, 46 patients and 47 lesions composed the matched cohort ( $\downarrow$ Table 3). Histological depth of invasion was significantly different before $(P=0.016)$ and after $(P=0.047)$ matching. Regarding curative resection, the distribution rate was not statistically different after matching $(P=0.254)$. On the other hand, after pairing, there were distinct means of lesion size between the two groups $(P=0.012)$. Detailed PSM evaluation results are shown in $>$ Appendix 1 and $>$ Appendix 2.

The median follow-up period for patients with HNSCC was 2.8 years and 3 years for patients without HNSCC. OS rates were similar in both groups ( $\mathbf{F i g . 2}$ ). In the HNSCC group, 10 deaths (21.2\%) were due to head and neck baseline cancer, on average 2 years after ESD. In the non-HNSCC, deaths were due to cardiovascular disease (2), 1 lung metastatic cancer (1), 1 liver hepatocellular carcinoma (1), progression of esophageal 


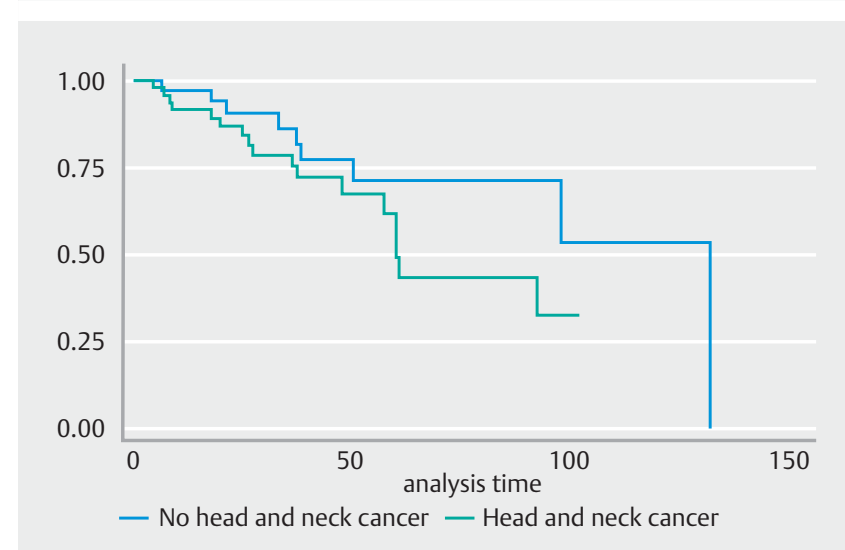

- Fig. 2 Overall survival rate. ${ }^{*} P(\log$ rank $)=0.146$

cancer (2) and unknown cause (3). Cancer-specific survival rates are shown in $\boldsymbol{\sim F i g .} \mathbf{3}$.

\section{Discussion}

ESCC accounts for about $90 \%$ of esophageal cancers worldwide. Regions of high incidence include Eastern to Central Asia, East Africa and South America. In general, rates in men exceed those of women and is mainly associated with cigarette smoking and alcohol consumption. These risk factors in common probably reflect the correlation between HNSCC and ESCC, explained by the concept of field cancerization, in which exposure to these carcinogens results in the development of multiple dysplastic and malignant lesions. In our study, the non-HNSCC group had a high ratio of female patients and lower intake of alcohol and smoking. This finding might highlight others risk factors and incidence patterns that are not yet entirely understood, for example. poor nutritional status, low intake of fruits and vegetables, drinking beverages at high temperatures, and infection with human papillomavirus.

The goal of endoscopic oncological therapy by means of ESD is complete removal of early stage disease. Although ESD has been widely used in Japan for ESCC, in other countries, it is still considered a cumbersome and challenging procedure with a steep learning curve and high risk of complications. In our study, rates of overall curative resection, en bloc resection, R0 resection, and AEs were $71.9 \%, 97.7 \%, 92.1 \%$ and $10.1 \%$, respectively, similar to other series of esophageal ESD [1719]. Based on these results, esophageal ESD seems to be feasible in Western centers with a high success rate.

Apparently, this is the first study aimed to compare esophageal ESD in patients with and without primary HNSCC. Our results suggest that HNSCC background does not adversely affect the clinical outcome of patients with superficial ESCC managed by ESD. OS and complication rates were similar between the two groups and consistent with findings from previous studies $[9,17,18,20-31]$.

Patients with HNSCC had ESCC detected at a younger age and more frequently consumed alcohol and smoked. The majority of neoplastic lesions were flat (Paris classification 0-IIB),

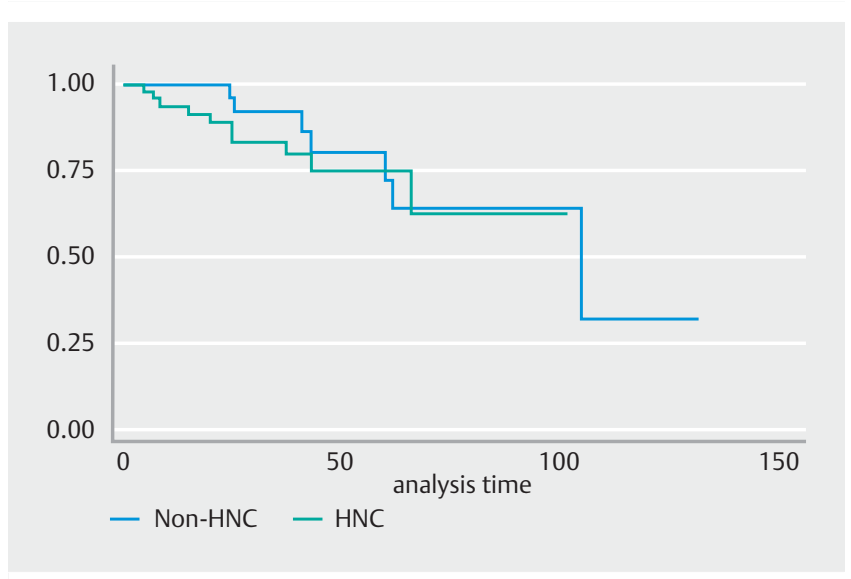

Fig. 3 Cancer-specific survival rate. ${ }^{*} P(\log$ rank $)=0.507$

which has a very low risk of deep SM invasion and is the most suitable morphological type to undergo endoscopic resection.

To reduce the impact of potential confounding factors and effects of selection bias, such as baseline clinicopathologic factors, PSM was applied to adjust the differences between the two groups. Results of histological invasion depth were consistent before and after PSM, which demonstrate that patients with HNSCC had ESCC detected at an earlier stage. However, the Paris classification was similar on the matched cohort ( $\triangleright$ Appendix 2), which can probably explain the similarity in curative resection rates after PSM. We speculated about possible reasons for the low curative resection rate $(57.8 \%)$ found in the non-HNSCC group.It is possible that the more advanced stage of the resected lesions could partially explain this suboptimal result in this group of patients. In fact, in the non-HNSCC group, there were more lesions that invaded deeper in the submucosa (>200 $\mu \mathrm{m})$, contributing to the lower curative resection rate observed in this group of patients.

Therefore, PSM indirectly supports that hypothesis that lesions detected in the high-risk group are more superficial and more likely to be cured with ESD. These findings may provide additional evidence that there is a beneficial effect of recommending screening endoscopy in this high-risk population and highlight the importance of systematic and carefully esophageal evaluation with virtual chromoendoscopy and lugol staining during endoscopy in patients with risk factors for esophageal neoplasms. Even though endoscopic resection indication criteria were the same between the two groups, in individuals HSNCC, esophageal lesions were more frequently shallow and confined into the mucosa layer. In our view, the lower curative resection rate observed in the non-HNSCC group can be partly explained by earlier diagnosis due to the effect of the screening program in patients with HNSCC.

The screening program might have prevented cancer-related mortality arising from the progress of ESCC in patients with HNSCC. Notwithstanding the above, there was no difference in OS. We hypothesize that the screening program may have affected ESCC-related mortality, but patients died mainly due to progression of head and neck disease. 
Even though previous chemoradiation (CRT) in theory may jeopardize ESD results due to fibrosis, with increased risk of severe $A E s$, we found no difference in ESD outcomes between the two groups, which is in accord with some studies that have demonstrated that ESD was technically feasible and safe despite CRT-induced fibrotic changes [32-35].

The current study is not without limitations. Because it was retrospective study, significant biases may have affected patient selection and caused misclassification. Data were obtained from both units' databases and, although entered in a prospective fashion, some follow-up information from a few patients was missing. The study was performed in two tertiary centers that specialize in cancer treatment, particularly for HNSCC management, which may have resulted in both performance and selection bias and may compromise external validation of the results. To better compare the groups in terms of potential confounding factors and reduce biases, we performed the PSM. However, it should be noted that many other variables were not included in the current study, which may have led to certain biases. Another limitation of this study refers to the indication for EGD in the groups being compared. In patients with HNSCC, EGD was indicated as part of a surveillance program for esophageal squamous cell cancer, while most patients without HNSCC had some kind of symptoms, mostly dyspeptic ones. Finally, because not all patients underwent exactly the same preoperative staging protocol, it is possible that this fact could partially explain the lower curative resection rate in the nonhead-and-neck cancer group.

\section{Conclusion}

In conclusion, patients with HNSCC have earlier detection of ESCC compared with sporadic detection in non-HNSCC, probably due to the endoscopic screening. Previous history of chemoradiation and surgery for HNSCC does not affect procedure time, AEs and OS.

\section{Competing interests}

The authors declare that they have no conflict of interest.

\section{References}

[1] Lim H, Kim DH, Jung HY et al. Clinical significance of early detection of esophageal cancer in patients with head and neck cancer. Gut Liver 2015; 9: 159-166

[2] Kim JS, Kim BW, Shin IS. Efficacy and safety of endoscopic submucosal dissection for superficial squamous esophageal neoplasia: A meta-analysis. Dig Dis Sci 2014; 59: 1862-1869

[3] Fukuhara T, Hiyama T, Tanaka S et al. Characteristics of Esophageal squamous cell carcinomas and Lugol-voiding lesions in patients with head and neck squamous cell carcinoma. J Clin Gastroenterol 2010; 44: e27-e33

[4] Watanabe S, Ogino I, Inayama Y et al. Impact of the early detection of esophageal neoplasms in hypopharyngeal cancer patients treated with concurrent chemoradiotherapy. Asia Pac J Clin Oncol 2017; 13: e3-e10
[5] Kandiah K, Chedgy FJQ, Subramaniam S et al. Early squamous neoplasia of the esophagus: The endoscopic approach to diagnosis and management. Saudi J Gastroenterol 2017; 23: 75-81

[6] Arantes V, Albuquerque W, Salles JMP et al. Effectiveness of unsedated transnasal endoscopy with white-light, flexible spectral imaging color enhancement, and lugol staining for esophageal cancer screening in high-risk patients. J Clin Gastroenterol 2013; 47: 314-321

[7] Ide E, Maluf-Filho F, Chaves DM et al. Narrow-band imaging without magnifcation for detecting early esophageal squamous cell carcinoma. World J Gastroenterol 2011; 17: 4408-4413

[8] Pimentel-Nunes P, Ponchon T, Repici A et al. Endoscopic submucosal dissection : European Society of Gastrointestinal Endoscopy ( ESGE) Guideline. Endoscopy 2015; 47: 829-854

[9] Ono S, Fujishiro M, Niimi K et al. Long-term outcomes of endoscopic submucosal dissection for superficial esophageal squamous cell neoplasms. Gastrointest Endosc 2009; 70: 860-866

[10] Rizvi Q ul ain, Balachandran A, Koay D et al. Endoscopic management of early esophagogastric cancer. Surg Oncol Clin N Am 2017; 26: 179191

[11] Yamamoto H, Oyama T, Gotoda T. Endoscopic submucosal dissection. esophageal cancer and Barrett's esophagus 2015: 177-188

[12] Arantes V, Albuquerque W, Freitas Dias CA et al. Standardized endoscopic submucosal tunnel dissection for management of early esophageal tumors (with video). Gastrointest Endosc 2013; 78: 946952

[13] Chow TL, Lee DTY, Choi CY et al. Prediction of simultaneous esophageal lesions in head and neck squamous cell carcinoma: A multivariate analysis. Arch Otolaryngol - Head Neck Surg 2009; 135: 882-885

[14] Rice TW, Blackstone EH, Rusch VW. Editorial: 7th edition of the AJCC cancer staging manual: Esophagus and esophagogastric junction. Ann Surg Oncol 2010; 17: 1721-1724

[15] Schlemper RJ, Riddell RH, Kato Y et al. The Vienna classification of gastrointestinal epithelial neoplasia. Gut 2000; 47: 251-255

[16] Kuwano H, Nishimura Y, Oyama T et al. Guidelines for Diagnosis and Treatment of Carcinoma of the Esophagus April 2012 edited by the Japan Esophageal Society. Esophagus 2015; 12: 1-30

[17] Chen Y, Zhao Y, Zhao X et al. Clinical outcomes of endoscopic submucosal dissection for early esophageal squamous cell neoplasms: a retrospective single-center study in China. Gastroenterol Res Pract 2016: 2016

[18] Toyonaga T, Man-I M, East JE et al. 1,635 endoscopic submucosal dissection cases in the esophagus, stomach, and colorectum: Complication rates and long-term outcomes. Surg Endosc 2013; 27: 10001008

[19] Fujinami H, Hosokawa A, Ogawa K et al. Endoscopic submucosal dissection for superficial esophageal neoplasms using the stag beetle knife. Dis Esophagus 2014; 27: 50-54

[20] Mochizuki Y, Saito Y, Tsujikawa T et al. Combination of endoscopic submucosal dissection and chemoradiation therapy for superficial esophageal squamous cell carcinoma with submucosal invasion. Exp Ther Med 2011; 2: 1065-1068

[21] Repici A, Hassan C, Carlino A et al. Endoscopic submucosal dissection in patients with early esophageal squamous cell carcinoma: results from a prospective Western series. Gastrointest Endosc 2010; 71: 715-721

[22] Teoh A, Chiu P, Ngo D et al. Outcomes of endoscopic submucosal dissection versus endoscopic mucosal resection in management of superficial squamous esophageal neoplasms outside Japan. J Clin Gastroenterol 2010; 44: e190-e194

[23] Yamashina T, Ishihara R, Uedo $\mathrm{N}$ et al. Safety and curative ability of endoscopic submucosal dissection for superficial esophageal cancers at least $50 \mathrm{~mm}$ in diameter. Gastroenterol Endosc 2014; 56: 515-521 
[24] Takahashi H, Arimura Y, Masao H et al. Endoscopic submucosal dissection is superior to conventional endoscopic resection as a curative treatment for early squamous cell carcinoma of the esophagus (with video). Gastrointest Endosc 2010; 72: 255-264

[25] Choi JY, Park YS, Jung HY et al. Feasibility of endoscopic resection in superficial esophageal squamous carcinoma. Gastrointest Endosc 2011; 73: 881-889

[26] Joo DC, Kim GH, Park DY et al. Long-term outcome after endoscopic submucosal dissection in patients with superficial esophageal squamous cell carcinoma: A single-center study. Gut Liver 2014; 8: 612618

[27] Park HC, Kim DH, Gong EJ et al. Ten-year experience of esophageal endoscopic submucosal dissection of superficial esophageal neoplasms in a single center. Korean J Intern Med 2016; 31: 1064-1072

[28] Youn YH, Park J, Park J] et al. Tu1580 clinical outcomes of endoscopic submucosal dissection for superficial esophageal squamous neoplasms. Gastrointest Endosc 2015; 81: AB517

[29] Probst A, Aust D, Markl B et al. Early esophageal cancer in Europe: Endoscopic treatment by endoscopic submucosal dissection. Endoscopy 2015; 47: 113-121
[30] Tsujii Y, Nishida T, Nishiyama O et al. Clinical outcomes of endoscopic submucosal dissection for superficial esophageal neoplasms: a multicenter retrospective cohort study. Endoscopy 2015; 47: 775-783

[31] Lee CT, Chang CY, Tai CM et al. Endoscopic submucosal dissection for early esophageal neoplasia: A single center experience in South Taiwan. J Formos Med Assoc 2012; 111: 132-139

[32] Nakahara T, Kushima R, Fujiyama Y et al. Chemoradiation therapy followed by endoscopic submucosal dissection for esophageal cancer. Dig Dis Sci 2008; 53: 3242-3245

[33] Nakajo K, Yoda Y, Hori K et al. Technical feasibility of endoscopic submucosal dissection for local failure after chemoradiotherapy or radiotherapy for esophageal squamous cell carcinoma. Gastrointest Endosc 2018; 88: 637-646

[34] Watanabe N, Jin M, Kimura Y et al. Salvage endoscopic submucosal dissection for the esophagus-localized recurrence of esophageal squamous cell cancer after definitive chemoradiotherapy. Gastrointest Endosc 2013; 79: 348-353

[35] Victor CR, Fujiki FK, Takeda FR et al. Safety and effectiveness of chemotherapy for metastatic esophageal cancer in a community hospital in Brazil. J Glob Oncol 2019: 1-10 
- Appendix 1 Patients characteristics after matching.

\begin{tabular}{|c|c|c|c|c|c|}
\hline & \multicolumn{2}{|c|}{ Group 1 (HNSCC) } & \multicolumn{2}{|c|}{ Group 2 (no-HNSCC) } & \multirow[t]{2}{*}{$P$} \\
\hline & $\mathbf{N}$ & $\%$ & $\mathbf{N}$ & $\%$ & \\
\hline Alcohol consumption & 46 & $100.0 \%$ & 46 & $100.0 \%$ & $1.000^{1}$ \\
\hline No & 4 & $8.7 \%$ & 4 & $8.7 \%$ & \\
\hline Yes & 42 & $91.3 \%$ & 42 & $91.3 \%$ & \\
\hline Smoking & 46 & $100.0 \%$ & 46 & $100.0 \%$ & $1.000^{1}$ \\
\hline No & 3 & $6.5 \%$ & 4 & $8.7 \%$ & \\
\hline Yes & 43 & $93.5 \%$ & 42 & $91.3 \%$ & \\
\hline Adjuvant therapy & 46 & $100.0 \%$ & 46 & $100.0 \%$ & 0.778 \\
\hline No & 39 & $84.8 \%$ & 38 & $82.6 \%$ & \\
\hline Yes & 7 & $15.2 \%$ & 8 & $17.4 \%$ & \\
\hline Mortality & 46 & $100.0 \%$ & 46 & $100.0 \%$ & 0.109 \\
\hline No & 29 & $63.0 \%$ & 36 & $78.3 \%$ & \\
\hline \multirow[t]{2}{*}{ Yes } & 17 & $37.0 \%$ & 10 & $21.7 \%$ & \\
\hline & \multicolumn{2}{|c|}{ mean $\pm S D$} & \multicolumn{2}{|c|}{ mean $\pm S D$} & \\
\hline Age (years) & \multicolumn{2}{|c|}{$59.2 \pm 9.41$} & \multicolumn{2}{|c|}{$58.74 \pm 9.82$} & $0,820^{2}$ \\
\hline $\begin{array}{l}\text { HNSCC, head and neck } s \\
P \text { in the Chi-squared test } \\
{ }^{1} \text { Fisher's exact test. } \\
{ }^{2} \text { Student's } t \text { test. }\end{array}$ & cinom & d deviation & & & \\
\hline
\end{tabular}

- Appendix 2 ESD outcomes after matching.

\begin{tabular}{|c|c|c|c|c|c|}
\hline & \multicolumn{2}{|c|}{ Group 1 (HNSCC) } & \multicolumn{2}{|c|}{ Group 2 (no-HNSCC) } & \multirow[t]{2}{*}{$P$} \\
\hline & $\mathbf{N}$ & $\%$ & $\mathbf{N}$ & $\%$ & \\
\hline Paris Classification & 47 & $100.0 \%$ & 47 & $100.0 \%$ & $0.621^{1}$ \\
\hline $0-\| \mathrm{A}$ & 4 & $8.5 \%$ & 2 & $4.3 \%$ & \\
\hline $0-11 \mathrm{~B}$ & 40 & $85.1 \%$ & 40 & $85.1 \%$ & \\
\hline $0-I I C$ & 3 & $6.4 \%$ & 5 & $10.6 \%$ & \\
\hline Circumference & 47 & $100.0 \%$ & 47 & $100.0 \%$ & $0.147^{1}$ \\
\hline$<50 \%$ & 13 & $27.7 \%$ & 14 & $29.8 \%$ & \\
\hline $50 \%-75 \%$ & 19 & $40.4 \%$ & 27 & $57.4 \%$ & \\
\hline $75 \%-100 \%$ & 12 & $25.5 \%$ & 5 & $10.6 \%$ & \\
\hline $100 \%$ & 3 & $6.4 \%$ & 1 & $2.1 \%$ & \\
\hline Histological depth of invasion & 47 & $100.0 \%$ & 47 & $100.0 \%$ & $0.047^{1}$ \\
\hline Intramucosa & 41 & $87.2 \%$ & 31 & $66.0 \%$ & \\
\hline Superficial submucosa & 5 & $10.6 \%$ & 11 & $23.4 \%$ & \\
\hline Deep submucosa & 1 & $2.1 \%$ & 5 & $10.6 \%$ & \\
\hline En bloc ressection & 47 & $100.0 \%$ & 47 & $100.0 \%$ & $1.000^{1}$ \\
\hline No & 1 & $2.1 \%$ & 0 & $0.0 \%$ & \\
\hline Yes & 46 & $97.9 \%$ & 47 & $100.0 \%$ & \\
\hline
\end{tabular}


-Appendix 2 (Continuation)

\begin{tabular}{|c|c|c|c|c|c|}
\hline & \multicolumn{2}{|c|}{ Group 1 (HNSCC) } & \multicolumn{2}{|c|}{ Group 2 (no-HNSCC) } & \multirow[t]{2}{*}{$P$} \\
\hline & $\mathbf{N}$ & $\%$ & $\mathbf{N}$ & $\%$ & \\
\hline R0 ressection & 47 & $100.0 \%$ & 47 & $100.0 \%$ & $0.677^{1}$ \\
\hline No & 4 & $8.5 \%$ & 2 & $4.3 \%$ & \\
\hline Yes & 43 & $91.5 \%$ & 45 & $95.7 \%$ & \\
\hline Curative resection & 47 & $100.0 \%$ & 47 & $100.0 \%$ & 0.254 \\
\hline No & 11 & $23.4 \%$ & 16 & $34.0 \%$ & \\
\hline Yes & 36 & $76.6 \%$ & 31 & $66.0 \%$ & \\
\hline \multicolumn{6}{|l|}{ Procedure-related complications } \\
\hline Bleeding & 46 & $100.0 \%$ & 47 & $100.0 \%$ & - \\
\hline No & 46 & $100.0 \%$ & 47 & $100.0 \%$ & \\
\hline Perforation & 47 & $100.0 \%$ & 47 & $100.0 \%$ & $0.495^{1}$ \\
\hline No & 45 & $95.7 \%$ & 47 & $100.0 \%$ & \\
\hline Yes & 2 & $4.3 \%$ & 0 & $0.0 \%$ & \\
\hline Stricture & 47 & $100.0 \%$ & 47 & $100.0 \%$ & 0.230 \\
\hline No & 33 & $70.2 \%$ & 38 & $80.9 \%$ & \\
\hline Yes & 14 & $29.8 \%$ & 9 & $19.1 \%$ & \\
\hline Local Recurrence & 47 & $100.0 \%$ & 47 & $100.0 \%$ & $1.000^{1}$ \\
\hline No & 46 & $97.9 \%$ & 47 & $100.0 \%$ & \\
\hline Yes & 1 & $2.1 \%$ & 0 & $0.0 \%$ & \\
\hline Metachronous tumor & 47 & $100.0 \%$ & 47 & $100.0 \%$ & 1.000 \\
\hline No & 38 & $80.9 \%$ & 38 & $80.9 \%$ & \\
\hline Yes & 9 & $19.1 \%$ & 9 & $19.1 \%$ & \\
\hline Synchronous tumor & 47 & $100.0 \%$ & 47 & $100.0 \%$ & 0.109 \\
\hline No & 44 & $93.6 \%$ & 39 & $83.0 \%$ & \\
\hline \multirow[t]{2}{*}{ Yes } & 3 & $6.4 \%$ & 8 & $17.0 \%$ & \\
\hline & \multicolumn{2}{|c|}{ mean $\pm S D$} & \multicolumn{2}{|c|}{ mean $\pm S D$} & \\
\hline Resected specimen size (mm) & \multicolumn{2}{|c|}{$31.26 \pm 16.89$} & \multicolumn{2}{|c|}{$23.04 \pm 7.99$} & $0.012^{2}$ \\
\hline Procedure time (min) & \multicolumn{2}{|c|}{$106.34 \pm 39.87$} & \multicolumn{2}{|c|}{$102.02 \pm 41.85$} & $0.610^{3}$ \\
\hline \multicolumn{6}{|c|}{$\begin{array}{l}\text { ESD, endoscopic submucosal dissection; HNSCC, head and neck squamous cell carcinoma; SD, standard deviation } \\
P \text { in the chi-squared test } \\
1 \text { Fisher's exact test. } \\
{ }^{2} \text { Mann-Whitney test. } \\
3 \text { Student's t test. }\end{array}$} \\
\hline
\end{tabular}

